\title{
Interest of $50 \%$ nitrous oxide and oxygen premix sedation in gerodontology
}

\author{
Emmanuel Nicolas 1,2 \\ Claire Lassauzay ${ }^{1,2}$ \\ ' $\mathrm{CHU}$ de Clermont-Ferrand, 63000 \\ Clermont-Ferrand, France; ${ }^{2}$ Université \\ Clermont I, EA 3847, Faculty of \\ Dentistry, 63000 Clermont-Ferrand, \\ France
}

\begin{abstract}
Elderly patients presenting cardiovascular, respiratory, or neurological disorders require a specific dental care approach, especially patients presenting Alzheimer's disease. Sedative procedures can prevent dental care-induced stress, even when there is effective pain control, but they have to be adapted to accommodate age-induced physiological modifications, age-related pathologies, and the concomitant treatments. In many situations, routine sedative prescriptions for dental care, such as benzodiazepine or antihistaminics, are not recommended for these patients. Nitrous oxide inhalation together with a specific behavioral threshold is currently the only sedative procedure adapted to cognitively-impaired elderly patients. Nitrous oxide is able to curb stress and its cardiovascular consequences, improve oxygenation, and optimize cooperation during dental care, making not only rehabilitation treatments but also routine dental care a viable option.
\end{abstract}

Keywords: nitrous oxide, oxygen, premix, sedation, gerodontology, dental care

\section{Introduction}

Medical procedures and public health systems need to adapt to the increasing needs of aging populations. The resulting problems are particularly relevant for oral health due to the age-induced increase in oral diseases. A French study ${ }^{1}$ on 756 institutionalized elderly subjects highlighted that $52 \%$ of the residents needed urgent dental care, whereas $89 \%$ of them were not currently receiving treatment. These needs are increased for subjects with Alzheimer's disease. Such patients have greater oral health needs ${ }^{2}$ as they present impaired oral hygiene, ${ }^{3}$ but have less oral care provision. ${ }^{4}$ Alzheimer's patients face social, ${ }^{5}$ economic, ${ }^{6,7}$ and technical obstacles to complete oral health: loss of cognitive and motor skills, loss of autonomy, physiological modifications, impaired adaptation processes, lack of compliance decreasing care access possibilities. Oral sphere alterations have been reported, ${ }^{2}$ such as impaired chewing capacity, impaired salivary flow, and problems swallowing. Normal aging is accompanied by progressive impairments of the cardiovascular and circulation systems ${ }^{8}$ characterized by adaptational difficulties in stressful situations (financial years, care), extremes for subjects with dementia. ${ }^{9}$ At the same time, intellectual agility decreases, and training processes and memory loss ${ }^{10}$ diminish the capacity to accept and adapt to change. These disturbances, which are recurrent in cognitively-impaired subjects, induce dental care problems. ${ }^{11,12}$

There has been relatively little research into dental management for uncooperative patients. The few articles that do consider specific oral rehabilitation for such patients suggest a dental procedure involving sedation or general anesthesia. ${ }^{13}$ However, sedation by inhalation of nitrous oxide/oxygen premix $\left(\mathrm{SPO}_{2}\right)$ remains a viable dental care strategy and represents the only alternative to general anaesthesia or abstention. There are many more indications covered ${ }^{14}$ than is possible under intravenous sedation. ${ }^{15}$ For younger subjects, the effectiveness and tolerance of $\mathrm{SPO}_{2}$ have been demonstrated $^{14,16}$ and the procedures validated, but for elderly subjects, the interest, tolerance and procedures have yet to be established. 


\section{Physiological context for elderly subjects during oral and dental care}

Normal aging is accompanied by a progressive reduction of respiratory system capacities. ${ }^{17,18}$ This, associated with impaired in-lung gaseous exchanges, explains the frequent hypoxemia encountered in elderly people. ${ }^{19}$ Respiratory control is also altered due to age, inducing decreased reactivity to hypoxemia and hypercapnea. ${ }^{20}$ Moreover, cough reflex and swallowing impairments ${ }^{21}$ can induce breathing disturbances. The impairments of the breathing system related to physiological aging commonly go undiagnosed and are brought to light in stress situations, meaning a prompt oxygen delivery is necessary, ${ }^{18}$ such as during impression-making for denture rehabilitation.

Similarly, normal aging is accompanied by progressive blood circulation impairments. ${ }^{8}$ At rest, the clinical consequences of these modifications remain minor for a long time, mostly involving only an increase in arterial pressure. However, the main characteristic of the elderly cardiovascular system is its difficulty with coping in stressful situations. ${ }^{18}$ Elderly people are particularly exposed during dental care procedures, and dental practitioners need to assess the risks and factor them into care. Although there is a known, real risk of cardiovascular accident during care, the consequences of a stress situation are often postponed, leading to increased blood pressure hours after care given. ${ }^{22}$

The dental care context can be considered as a factor of stress and of cardiovascular effort. Simply evoking the dental treatment can be enough to induce an increase in heart rate and muscular tension. ${ }^{23,24}$ Studies have been performed to evaluate the stress provoked by dental care in elderly subjects. ${ }^{25} \mathrm{~A}$ recent study highlighted the age-related differences in variations of physiological parameters such as heart rate, blood pressure, and blood saturation of oxygen during an impression-making session. ${ }^{22}$ The elderly reacted more vigorously to the stress caused by impression-making than younger subjects, and their parameters were slower to return to normal. The variation of these physiological parameters, when extreme, can put vital functions at risk. Dental practitioners, during their interventions, should therefore take these modifications into account, and continuously monitor pulse oxymetry, prevent hypoxemia by facilitating breathing, and initiate oxygen therapy if necessary, with protection of airways.

\section{Proposals for coping with the impaired cardiovascular adaptability of elderly patients}

Impaired cardiovascular adaptability means that dental care-induced stress needs to be controlled. In this context, behavioral management is helpful in limiting stress, fostering compliance and enhancing cooperation during care. The techniques need to be geared to age and cognitive deficiency. Thus, precepts of the communication and behavior management strategies can be employed with the persons presenting cognitive deficit. ${ }^{26}$ This makes initial hospitality a key moment in the care procedure, since it is based on concepts of respect, dignity and empathy. Appointments should be planned in the late morning in order to avoid changing waking and breakfast habits, or in the early afternoon. Care sessions late in the afternoon are not indicated because many dementia patients are known to show behavioral alterations and experience anxiety linked to nightfall ('sundown syndrome'). The elderly present auditory, visual and/or cognitive deficiencies that can lead to stressful situations when they are exposed to overwhelming stimulus in an unfamiliar environment. Thus, to deal with decreased visual acuteness and lighting acclimatization, the practitioner should ideally be situated in front of the patient, fully in their line of sight, to avoid the dazzle caused by the dental chair light. Impaired auditory capability must also be considered, as low frequency sounds are perceived in an abnormally strong way. It is therefore necessary to speak loudly, but without shouting, articulating patiently, and avoiding any sudden noise or breaks. Verbal communication has to remain uninterrupted throughout the session. The care procedure has to be explained and repeated to the patient throughout the session, even at late stage of dementia, if there is any persistent doubt as to level of understanding. During care, it is important to repeat encouragements to avoid feelings of failure and to reassure the patient. The sentences and words employed must be simple, and punctuated by breaks, while explanations have to be reassuring but not infantilizing. Nonverbal communication, by physical contact (hands held for example) or visual contact (looks, smiles), is also an efficient means of comforting the patient. Whether it is by verbal or physical channels, the patient must be made aware of the practitioner's empathy and respect. When cognitive-behavioral techniques alone are unable to manage the patient's stress, the indication becomes to implement a sedative pre-treatment or to realize the care under sedation.

\section{Age-related psychological and psychiatric features for oral health and dental care}

Cooperation and compliance are decision-making factors in dental treatment planning. In the absence of associated 
pathology, most elderly maintain their intellectual capacities and creativity. However, after 70 years of age, decreases in intellectual agility as well as some deterioration in training processes and in memory ${ }^{10}$ are reported. Elderly subjects therefore experience more difficulties in dealing with unusual situations, which can lead to withdrawal and social isolation. Around $15 \%-30 \%$ of people aged 65 and over experience significant depressive symptoms. In institutional care, the prevalence of depression is about $40 \%$. Stress and anxiety are omnipresent during care, regardless of age. In this situation, pain during care can generate anxiety and care avoidance, which aggravate difficulties in integrating the healthcare system. ${ }^{27}$ Consequently, these subjects show an increased prevalence of infectious oral pathologies like decay and periodontal disease. ${ }^{28}$ This disadvantage is a public health concern, involving $10 \%-18 \%$ of Europeans and $19 \%$ of North Americans. ${ }^{29}$ Prevalence has not been widely studied in elderly people, but is cited at $8.4 \%$ in elderly Canadian subjects, ${ }^{30} 10 \%$ in Swedish women aged 60 and over $^{31}$ and $7.6 \%$ of French persons aged 65 and over. ${ }^{32}$

\section{Problems linked to cognitive impairment}

The dental state of subjects presenting dementia is commonly aggravated by illness, ${ }^{33,34}$ making dental care difficult. Independently of the presence of dental phobia or dental anxiety, there is greater difficulty with cooperation with a background of dementia. Pain evaluation for cognitively-impaired subjects is a problem for the practitioner. The pain experienced by elderly subjects is underestimated; hence the risk of not diagnosing pain is higher for subjects presenting cognitive disturbances, ${ }^{35,36}$ due to the subjects' inability to express pain, absence of anatomo-clinical parallelism and large inter-subject variability. Pain evaluation thus has to be based on observation in the daily monitoring of parameters such as sudden reduction of motility, or behavioral or feeding disturbances. These modifications are more visible by family or carers, but not in a routine way by dentists.

\section{Proposal for uncooperative elderly patients: sedation procedure by inhalation of MEOPA}

\section{General framework for use}

When the subject is unable to cooperate to the required level, sedation by inhalation of nitrous oxide/oxygen premix offers an alternative to general anesthesia that covers more indications than is possible under heavier sedations administered by intravenous route. ${ }^{15}$ The pharmacological action is identical to morphine derivatives. The $50 \%$ gas mix allows sedation (inhibition of gag reflex and involuntary movements) and analgesia (covering the pain of anesthetic injections). Nitrous oxide has low blood solubility but diffuses rapidly across the alveolar-arterial membrane. Consequently, induction of sedation and recovery are both very quick, gas metabolism is negligible and almost exclusively eliminated through the lungs. This sedation format does present some absolute contraindications, such as closed air cavities, recent cranial traumatism, intracranial hypertension, and consciousness disturbances, plus other relative contraindications such as cardiac or deep broncho-pulmonary insufficiency, facial traumatism, or intervention site interfering with the mask application region. Most studies report the use of $50 \%$ nitrous oxide/ oxygen mixture in combination with other medications. ${ }^{14}$ So, precautionary measures need to be taken if the patient is using medicines that alter consciousness or breathing patterns (ie, morphine derivatives, benzodiazepines, and other psychotropics). Nevertheless, no study has been yet conducted in geriatrics.

\section{Effectiveness and tolerance in geriatric subjects}

There are very few studies specifically targeting the effectiveness or tolerance of sedation techniques in the elderly, although the topic is widely described for children and adults. The criteria widely taken in account when studying the effectiveness of nitrous oxide are the feasibility of the procedure and/or patient cooperation. Reported success rates range from $64 \%{ }^{37}$ to $94 \%{ }^{38}$ when administered during urgent care, ${ }^{39}$ in pediatric procedures $^{40}$ and in gynecological procedures. ${ }^{41}$ In dentistry, the effectiveness of sedation is cited at around $90 \%,{ }^{42,43}$ but this figure did not exclusively cover patients aged 65 and over.

In a review by Collado and colleagues, ${ }^{42}$ the prevalence of minor adverse events during sedation with nitrous oxide was reported as ranging from $0 \%{ }^{44}$ to $68 \%{ }^{45}$ according to the indications. Onody and Hennequin, ${ }^{46}$ studying 35,828 sessions with oxygen/nitrous oxide premix, reported a $4.4 \%$ rate of adverse effects, $45 \%$ of which were gastrointestinal reactions (nausea, vomiting), 41\% neurological (headache, vertigo) and $14 \%$ not specified. This study assessed the risk of adverse effects at 3/10,000 administrations. One study on elderly patients ${ }^{47}$ focusing on tolerance of nitrous oxide when 
used for analgesia recorded $22 \%$ adverse effects, all minor. Another study on 82 dental care sessions accomplished by practitioners newly trained in this sedation technique ${ }^{43}$ evaluated the rate of adverse effects during geriatric dental care as $17 \%$.

\section{Nitrous oxide sedation in gerodontology}

In theory, all types of dental care (hygiene, conservative care, surgery, implants, and prosthesis) are possible under sedation by inhalation of nitrous oxide. The difficulty resides more in the number of sessions necessary to complete the oral treatment. The organization necessary for transport, accompanying carers when needed, and patient tiredness caused by the care process and travel makes a long series of sessions impracticable. The therapy must therefore be adapted to the number of possible sessions. At the first stage, most acts accomplished under sedation for resistive or uncooperative patients are centered on oral hygiene. Oral hygiene restores oral comfort and decreases the risks of infectious respiratory tract diseases such as pneumonia, which is the major cause of hospital fatalities in elderly patients. Sedation by inhalation can be regularly repeated to clean up the oral cavity of patients resisting hygiene care. These elements are particularly important for immunodepressed patients or patients presenting a depressed cough reflex. The concept of the benefit/risk ratio justifies that avulsions are the most frequent acts. For elderly patients presenting swallowing impairments, sutures after avulsion in order to avoid blood and saliva being inhaled are easier under sedation. Either way, prosthodontic rehabilitation plans remain simple.

\section{Interest of nitrous oxide in dementia settings}

The interest of sedation by inhalation of nitrous oxide in dentistry has been assessed for dental care-phobic patients, young children, subjects with mental deficiencies and some spot indications (gag reflex). The sedative effect of the nitrogen protoxide can be also useful for elderly subjects presenting cognitive disturbances. In most cases, the opposition behavior of these patients is directly linked to the absence of dental care. Infections and/or painful states set in, but sedative, analgesic and antibiotic medication are not provided due to difficulties of expression and perception in neurodegenerative pathology. This means that the clinician faces the risk of underestimating the patient's needs, and it has been reported that dementia patients use less pain-relieving sedative analgesics than noncognitively-impaired subjects. ${ }^{48}$

\section{Description of dental care sessions with nitrous oxide}

During the care session, two practitioners have to be present. One of the carers practises the care, meanwhile the other surveys patient's physiologic parameters (blood pressure, heart rate, and blood oxygenation), and state of sedation, patient skin color and sudation. All along the gas administration, breathing is surveyed by the balloon movements of inflation/deflation.

Primary to sedation, the events expected to occur during the session are explained to the patient, even if his cognitive skills are deeply impaired, then the mask positioned on his nose and mouth, and its utility explained. Then sedation can begin, the induction duration, usually realized in three minutes for adults, is longer and subject to variation in elderly. Light physical restraint is sometimes necessary at the beginning of induction and usually realized by holding hands away from the mask, and when possible, by the patient's accompanying person. All along the session, the practitioners keep explaining calmly what is done, and reassuring the patient. Completion of sedation is sometimes difficult to assess with patient presenting Alzheimer disease, but usually, when neuromotor disorders cease (teeth grinding, hands tension), induction is completed. Dental care with such type of sedation has to be no longer than 30 minutes, and the less tiring for the patient as possible. Prior to the sedation session, the acts of care are determined but then the treatment is adjusted to the behavior and the physical parameters of the patient. The care can begin after the induction is completed by removing the mask from the nose and mouth of the patient. In children and cooperative adults the mask is usually put on the nose and the patient asked to breath by the nose. For uncooperative subjects, nose respiration on purpose is impossible so there is no utility to put the mask in this position which encroaches on their vision, and can lead to behavioral disorders. The time intend for care between two applications of mask is short, about 30 to 45 seconds and the practitioner must have clinical experience in providing dental care under this type of sedation to be efficient. The duration of recovery after sedation depends on the patient breathing capacity (about $3 \mathrm{mn}$ ). A diminution in blood oxygen saturation can occur just after the mask removal, but necessity of oxygen inhalation remains rare. The session is completed when the patient regains his skills, for Alzheimer's patients, the recovery of babble or stereotyped movements (scratching, rubbing, teeth grinding) sign recuperation. 


\section{Conclusion}

In settings where patients display a high level of opposition to treatment, sedation by nitrous oxide appears to be a good alternative to general anesthesia. ${ }^{49}$ Clinical procedure for these frail patients is a difficult choice, and the notion of benefit/risk ratio has to be taken into account. There is a real risk of complications under general anesthesia, especially for this type of patient. However, the tolerance and effectiveness of nitrous oxide in these subjects remains to be confirmed through clinical trials. In all situations, ethically-speaking, it is difficult to leave elderly patients exposed to such infectious situations, with all concomitant consequences (pain, risk of breathing in germs, septicemia, and heart disorder).The decision must therefore be made collegially, with the family and the anesthetist, according to the clinical situations and the presence of co-morbidities.

\section{Disclosure}

The authors report no conflicts of interest in this work.

\section{References}

1. Cohen C, Tabarly P, Hourcade S, Kirchner-Bianchi C, Hennequin M. [Oral hygiene for the institutionalized elderly]. Presse Med. 2006;35 (11 Pt 1):1639-1648.

2. Roberts WE, Garetto LP, Arbuckle GR, Simmons KE, DeCastro RA. What are the risk factors of osteoporosis? Assessing bone health. J Am Dent Assoc. 1991;122:59-61.

3. Sreebny LM, Schwartz SS. A reference guide to drugs and dry mouth. Gerodontology. 1986;5:75-99.

4. Gilbert GH, Branch LG, Orav EJ. Predictors of older adults' longitudinal dental care use. Ten-year results. Med Care. 1990;28:1165-1180.

5. Blanco VL, Levy SM, Ettinger RL, Logan H, Buckwalter KC. Challenges in geriatric oral health research methodology concerning caregivers of cognitively impaired elderly adults. Spec Care Dentist. 1997; 17:129-132.

6. Ship JA, Puckett SA. Longitudinal study on oral health in subjects with Alzheimer's disease. J Am Geriatr Soc. 1994;42:57-63.

7. Chapman PJ, Shaw RM. Normative dental treatment needs of Alzheimer patients. Aust Dent J. 1991;36:141-144.

8. Weisfeld, ML. Lakatta EG, Gerstenblith G. Aging and cardiac disease. In: Braunwald E, editor. Braunwald's Heart Disease: A Textbook of Cardiovascular Medicine. Philadelphia, PA: WB Saunders; 1990. p. 1560-1562.

9. Rosendorff C, Beeri MS, Silverman JM. Cardiovascular risk factors for Alzheimer's disease. Am J Geriatr Cardiol. 2007;16:143-149.

10. Masoro EJ. Physiology of aging. Int J Sport Nutr Exerc Metab. 2001;11(Suppl):S218-222.

11. Kieser J, Jones G, Borlase G, MacFadyen E. Dental treatment of patients with neurodegenerative disease. N Z Dent J. 1999;95:130-134.

12. Nordenram G, Ryd-Kjellen E, Ericsson K, Winblad B. Dental management of Alzheimer patients. A predictive test of dental cooperation in individualized treatment planning. Acta Odontol Scand. 1997;55:148-154.

13. Fiske J, Frenkel H, Griffiths J, Jones V. Guidelines for the development of local standards of oral health care for people with dementia. Gerodontology. 2006;23(Suppl 1):5-32.
14. Hennequin M, Maniere MC, Albecker-Grappe S, et al. A prospective multicentric trial for effectiveness and tolerance of a $\mathrm{N}_{2} \mathrm{O} / \mathrm{O}_{2}$ premix used as a sedative drug. J Clin Psychopharmacol. 2004;24:552-554.

15. Malamed SF, Quinn CL. Sedation: a guide to patient management. Saint-Louis, MO: Mosby; 2003.

16. Peretz B, Katz J, Zilburg I, Shemer J. Response to nitrous-oxide and oxygen among dental phobic patients. Int Dent J. 1998;48:17-23.

17. Chen HI, Kuo CS. Relationship between respiratory muscle function and age, sex, and other factors. J Appl Physiol. 1989;66:943-948.

18. Juvin P, Brion F, Teissiere F, Durigon M. Prolonged activity after an ultimately fatal gunshot wound to the heart: case report. Am J Forensic Med Pathol. 1999;20:10-12.

19. Wahba WM. Influence of aging on lung function-clinical significance of changes from age twenty. Anesth Analg. 1983;62:764-776.

20. Peterson DD, Pack AI, Silage DA, Fishman AP. Effects of aging on ventilatory and occlusion pressure responses to hypoxia and hypercapnia. Am Rev Respir Dis. 1981;124:387-391.

21. Pontoppidan H, Beecher HK. Progressive loss of protective reflexes in the airway with the advance of age. JAMA. 1960;174:2209-2213.

22. Nicolas E, Lassauzay C, Pickering G, Croze J, Hennequin M. Needs in screening cardiovascular parameters during dental care in the elderly. Aging Clin Exp Res. 2008;20:272-276.

23. Johnsen BH, Thayer JF, Laberg JC, et al. Attentional and physiological characteristics of patients with dental anxiety. $J$ Anxiety Disord. 2003; 17:75-87.

24. Lundgren J, Berggren U, Carlsson SG. Psychophysiological reactions in dental phobic patients during video stimulation. Eur J Oral Sci. 2001;109:172-177.

25. Bedi R, McGrath C. Factors associated with dental anxiety among older people in Britain. Gerodontology. 2000;17:97-103.

26. Chalmers JM. Conducting geriatric dental research with cognitively impaired adults. Gerodontology. 2000;17:17-24.

27. Berggren U, Meynert G. Dental fear and avoidance: causes, symptoms, and consequences. J Am Dent Assoc. 1984;109:247-251.

28. Armfield JM, Stewart JF, Spencer AJ. The vicious cycle of dental fear: exploring the interplay between oral health, service utilization and dental fear. BMC Oral Health. 2007;7:1.

29. Locker D, Ford J, Leake JL. Incidence of and risk factors for tooth loss in a population of older Canadians. J Dent Res. 1996;75:783-789.

30. Locker D, Liddell A, Burman D. Dental fear and anxiety in an older adult population. Community Dent Oral Epidemiol. 1991;19:120-124.

31. Hagglin C, Hakeberg M, Hallstrom T, et al. Dental anxiety in relation to mental health and personality factors. A longitudinal study of middleaged and elderly women. Eur J Oral Sci. 2001;109:27-33.

32. Nicolas E, Collado V, Faulks D, Bullier B, Hennequin M. A national cross-sectional survey of dental anxiety in the French adult population. BMC Oral Health. 2007;7:12.

33. Ghezzi EM, Ship JA. Systemic diseases and their treatments in the elderly: impact on oral health. J Public Health Dent. 2000;60:289-296.

34. Ghezzi EM, Ship JA. Dementia and oral health. Oral Surg Oral Med Oral Pathol Oral Radiol Endod. 2000;89:2-5.

35. Ferrell BA. Pain management in elderly people. J Am Geriatr Soc. 1991;39:64-73.

36. Sengstaken EA, King SA. The problems of pain and its detection among geriatric nursing home residents. $J$ Am Geriatr Soc. 1993;41:541-544.

37. Warren VN, Crawford AN, Young TM. The use of Entonox as a sedation agent for children who have refused operative dentistry. J Dent. 1983;11:306-312.

38. Hallonsten AL, Koch G, Schroder U. Nitrous oxide-oxygen sedation in dental care. Community Dent Oral Epidemiol. 1983;11:347-355.

39. Oji A, Thakran RS, Thakran PC. Entonox for casualties at $1000 \mathrm{~m}$ : use of nitrous oxide analgesia in Nigeria at moderate altitude. Anaesthesia. 1984;39:1127-1130.

40. Harrop JE. Management of pain in childhood. Arch Dis Child Educ Pract Ed. 2007;92:ep101-108. 
41. Volmanen P, Akural E, Raudaskoski T, Ohtonen P, Alahuhta S. Comparison of remifentanil and nitrous oxide in labour analgesia. Acta Anaesthesiol Scand. 2005;49:453-458.

42. Collado V, Nicolas E, Faulks D, Hennequin M. A review of the safety of 50\% nitrous oxide/oxygen in conscious sedation. Expert Opin Drug Saf. 2007;6:559-571.

43. Collado V, Nicolas E, Faulks D, et al. Evaluation of safe and effective administration of nitrous oxide after a postgraduate training course. BMC Clin Pharmacol. 2008;8:3.

44. Douard MC, di Palma M, d'Agostino P, et al. Prospective, double-blind, randomized trial of equimolar mixture of nitrous oxide/oxygen to prevent pain induced by insertion of venous access ports in cancer patients. Support Care Cancer. 2006;14:161-166.

45. Wang CY, Chiu CL, Har KO, Chan C, Rahman ZA. A comparative study of sevoflurane sedation with nitrous oxide sedation for dental surgery. Int J Oral Maxillofac Surg. 2002;31:506-510.
46. Onody P, Gil P, Hennequin M. Safety of inhalation of a $50 \%$ nitrous oxide/oxygen premix: a prospective survey of 35828 administrations. Drug Saf. 2006;29:633-640.

47. Bauer C, Lahjibi-Paulet H, Somme D, Onody P, Saint Jean O, Gisselbrecht M. Tolerability of an equimolar mix of nitrous oxide and oxygen during painful procedures in very elderly patients. Drugs Aging. 2007;24:501-507.

48. Pickering G, Jourdan D, Eschalier A, Dubray C. Impact of age, gender and cognitive functioning on pain perception. Gerontology. 2002;48:112-118

49. Lopez-Jimenez J, Gimenez Prats MJ. Sedation in the geriatric patient. Med Oral. 2004;9:45-55. 